\title{
Theoretical Analysis of Magnetohydrodynamical Waves for Plasma based Coating Process of Isothermal Viscous-Plastic Fluid
}

\author{
UMER REHMAN ${ }^{1, *}$, MUHAMMAD BILAL ${ }^{2}$, MUBBASHIR SHERAZ ${ }^{2}$ \\ ${ }^{1}$ Department of Physics, Air University E-09 Sector Islamabad 44000, PAKISTAN \\ ${ }^{2}$ Department of Mathematics, University of Wah Rawalpindi, PAKISTAN
}

\begin{abstract}
A mathematical formulation on coating of a thin film for a compressible isothermal magnetohydrodynamic (MHD) viscous-plastic fluid flowing across a narrow gap between two rotating rolls is described in this article. The lubrication approximation theory is used to create and simplify the equations of motion required for the fluid injected for coating. The relation explaining MHD wave dynamics and instability is obtained by analytical calculations. According to the current investigation, the growth rate in the unstable MHD waves are numerically evaluated as the function of the concerned parameters, It's worth noting that the Lundquist and Prandtl's numbers are growth rate control parameters in unstable MHD modes. The results show that the viscous-plastic parameter and ratio of diffusion rate have a significant impact on the fundamental MHD dynamics. It is also concluded that the MHD effects have had a significant impact on the coating of Casson material.
\end{abstract}

Keyword: MHD waves, Viscous-Plastic Plasma Fluid, MHD theory, Coating

Received: June 13, 2021. Revised: January 4, 2022. Accepted: January 25, 2022. Published: February 21, 2022.

\section{Introduction}

Surface coating using a fluid film is common in the magnetic tape, photographic, wrapping industries, polymer sheet, hot rolling, glass-fabric, wire drawing, as well as for manufacturer of paint and paper, to protect a large surface region with one/many uniform layers [1-4]. Despite the fact that the products utilized in various coating sectors vary greatly, while the same basic technologies are used to create the necessary coatings. A fluid deposited film should be thin, continuous, and homogeneous in thickness in general. Of contrast, instabilities in films are detected under particular operating conditions, which can only be investigated by delving into the challenges of fluid dynamics associated to the coating process, as demonstrated in the approach $[5,6]$ for Newtonian flows.

The concept of MHD threedimensional Casson fluid across a porous linear stretching sheet was presented by Mahanta et al. [7]. Later, Mustafa et al. [8] investigated boundary layer flow for a Casson nano fluid using a nonlinear stretching surface. The boundary layer flow for a steady incompressible laminar free convective MHD Casson fluid across an exponential stretching surface grounded in a thermal stratified medium was examined by Animasaun et al. [9]. Further, Das et al. [10] investigated the influence of mass and heat transmission in a vertical plate for an unstable Casson fluid. In physics, chemistry, and engineering, the study of boundary layer 
flow across a continually extending sheet has practical applications. Many metallurgical processes, such as plastic film drawing, annealing, copper wire thinning, etc, play a vital role in managing momentum and heat transfer boundary layer flow for a stretching sheet. In the applied magnetic field, Ganesh et al. [11] examined the numerical solution for nano-fluid across a linearly semi-infinite stretched sheet. Dessie et al. [12] proposed the MHD flow theory for incompressible viscous fluid and heat transfer phenomena over a stretching sheet embedded in porous medium with heat source/sink and viscous dissipation. The effect of partial slip on hydro-magnetic boundary layer flow and heat transfer due to stretched surface with thermal radiation was investigated by Hakeem et al. [13]. For better understand of MHD fluid flow, Hayat et al. [14] studied a hydromagnetic thirdgrade fluid across a continually stretched cylinder and used a homotopic technique to solve the problem. The approximate solution for MHD flow of tangent hyperbolic fluid model on a stretching cylinder was investigated by Malik et al. [15]. The idea of two-dimensional MHD radiative fluxes and heat transmission of a dusty nano fluid over an exponentially extending surface was discussed by Sandeep et al. [16]. From published literature it is observed that steady MHD wave dynamics and instabilities of Casson fluid over a stretching sheet has not been discussed so far. In Maxwellian fluid dynamics, nonetheless, MHD waves and instabilities remain to be of key relevance. This issue has been addressed in current investigation.
Although, the roll coating procedure is simulated in this theoretical work to categorize the characteristic time and length scale associated with MHD wave dynamics, nevertheless the formulation is applicable to Casson charged particle fluid in any plasmabased process in the presence of an externally applied magnetic field. Roll coating is a diverse procedure that requires technical and operational expertise to maintain consistency. The use of a coated steel strip in the manufacturing industry is currently expanding to include more novel features such as acoustical protection coatings and photovoltaic coatings that absorb solar energy [17]. In recent decades, experimental studies, theoretical approaches, and numerical analysis have all been used to investigate the flow challenges of roll coating processes. The necessity in coating manufacturing to coat stably, quickly, and uniformly thin layers by improving coater operating parameters and coating microstructure spurred the work's innovation. When both the roll and the sheet are moving at the same speed, Zafar et al. [18] discussed the roll-coating analysis of an incompressible nanofluid. Using lubrication approximation theory, the simplified form of the equation of motion was developed. Recently, Zheng et al. [19] explored reverse roll coating of non-isothermal MHD viscoplastic fluid theoretically. To the best of our understanding and belief, there is no reference in the theoretical formulation on MHD wave's dynamics and associated instabilities for roll coating with the Casson fluid model. The aim of this research is to develop a mathematical model for MHD flow instabilities in viscous-plastic fluids so 
that key MHD waves can be studied theoretically in the presence of an external magnetic field.

The rest of manuscript is arranged in the following manner. Section 2 is apportioned for model and governing equations, in the section normal mode analysis has been carried out. In Section 3 results have been interpreted and discussion on relevant physics has been presented. Section 4 contains brief summary of the work.

\section{The Model and Governing Equations}

Assume a laminar, and a compressible isothermal flow of viscous-plastic MHD fluid in externally applied magnetic field between two rolls revolving in opposite directions with velocities $V_{f r}=$ $\omega_{f r} r$ and $V_{r v}=\omega_{r v} r$, respectively, where $r$ is the radius of each roll and the subscripts $f r$ and $r v$ denote forward and reverse rotating rolls. Applied magnetic field $B=B_{0} \hat{x}$ is along $\mathrm{x}$-direction in rectangular coordinate system. The nip area is the shortest distance between two revolving rollers, and the range at the nip region is denoted by $2 H_{0}$. Moreover, the $\mathrm{x}$-direction is taken parallel to the flow movement, whereas the y-direction is taken perpendicular to the direction of flow, as shown in Figure 1.

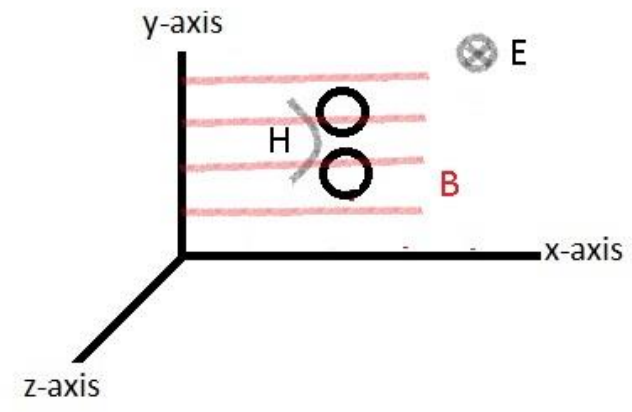

Figure 1. Geometry and schematic representation of the process under consideration.

The mathematical model for geometry described above is presented, and it is based on a set of equations as shown below:

$$
\rho\left(\frac{\partial v}{\partial t}+v \cdot \nabla v\right)=-\nabla p+\boldsymbol{j} \times \boldsymbol{B}+
$$

$\nabla \cdot \tau$

$$
\frac{\partial n}{\partial t}+\nabla \cdot(n \boldsymbol{v})=0
$$

$$
\boldsymbol{E}=-\boldsymbol{v} \times \boldsymbol{B}+\eta \boldsymbol{j}
$$

Equation (1) is the momentum conservation, Equation (2) is the mass conservation and Equation (3) is the Ohm's law for the Maxwellian fluid. Where $v$ is the velocity of the fluid; $\rho$ is the mass density; $p=n K T$ is the pressure ( $T$ is constant for isothermal fluid ); $\boldsymbol{j}$ is the current density; $\boldsymbol{E}$ is the electric field; the magnetic field $\boldsymbol{B}=$ $\boldsymbol{B}_{\boldsymbol{0}}+\boldsymbol{b}$ composed of externally applied and induced component, respectively; $\eta$ is the fluid's electric resistivity; and the stress tensor is represented by $\tau$ for the fluid model $[20,21]$. The equation of the stress tensor composed of two parts gyro-viscous part $\nabla$. 
$\tau_{g y r}=-\rho v \cdot \nabla v$ and isotropic part $\nabla \cdot$ $\tau_{\text {iso }}=-\mu_{B} \nabla^{2} v-\left(2 \mu_{c}-\mu_{B}\right) \nabla(\nabla \cdot v)$,

fluids. Deformation rate based on the model of non-Newtonian fluid, so $\mu_{c}$ is the critical value of off diagonal product terms.

In two-dimensional flow for rectangular coordinate system, we use the formula as follows:

$$
v=[U(x, y), V(x, y)]
$$

The Lorentz force $\boldsymbol{j} \times \boldsymbol{B}$ becomes $\frac{1}{\eta}(\boldsymbol{E}+$ $\boldsymbol{v} \times \boldsymbol{B}) \times \boldsymbol{B}$ from Equation (3). The Lorentz force becomes: in this circumstance, due to the magnetic field:

$$
\boldsymbol{j} \times \boldsymbol{B}=-\frac{2 B_{0}^{2} v}{\eta}
$$

Where triple vector product identity is used along with drift velocity relation $\boldsymbol{v}=$ $\boldsymbol{E} \times \boldsymbol{B} / B_{0}^{2}$. Equations (1) can be expressed as follows using Equations (2), (4), and (5):

$$
\begin{gathered}
\rho\left(\frac{\partial U}{\partial t}+U \frac{\partial U}{\partial x}+V \frac{\partial U}{\partial y}\right)=-\frac{\partial(n K T)}{\partial x}- \\
\frac{2 B_{0}^{2} U}{\eta}+\frac{\partial \tau_{x x}}{\partial x}+\frac{\partial \tau_{x y}}{\partial y} \\
\rho\left(\frac{\partial V}{\partial t}+U \frac{\partial V}{\partial x}+V \frac{\partial V}{\partial y}\right)=-\frac{\partial p}{\partial y}- \\
\frac{2 B_{0}^{2} V}{\eta}+\frac{\partial \tau_{x x}}{\partial x}+\frac{\partial \tau_{y y}}{\partial y}
\end{gathered}
$$

These are the equations for dynamical Casson fluid flow retains all effect of electric and magnetic force in the dynamics of fluid, in the case of incompressible steady where $\mu_{B}$ represents the viscosity of plastic properties for non-Newtonian state flow with zero electric field and very small induced magnetic field compared to the applied magnetic field, so that the magnetic Reynolds number is negligible, set of equation given in Ref. [22] are retrieved. The most significant dynamic phenomenon occurs in the roll coating at the nip, according to the problem's geometry. $2 \mathrm{H}_{0}$ between rolls characterizes the smallest gap at the nip region. As a result, it may be advantageous to suppose that the flows are virtually parallel, with the common fluid motion largely in the $\mathrm{x}$-direction and the fluid's minimal velocity in the y-direction. Additionally, the y-direction velocity increase takes precedence over the $\mathrm{x}$ direction flow velocity. To make things easier, we can use an order of magnitude analysis to determine the scale of pressure and velocity parameters, identifying $U, \mathrm{x}$, and $\mathrm{y} \quad$ as $U \sim u, \quad x \sim L, \quad$ and $y \sim H_{0}$, respectively. In view of these relations, we arrive at $H_{0} / L \ll 1$, where $L=\sqrt{H_{0} r}$, hence Equation (6) can be written as;

$$
\begin{aligned}
& \rho T \frac{\partial n}{\partial x} \\
& \rho t
\end{aligned}
$$

In Equation (8) gyro-viscous cancellation has been incorporated as given in Ref. [20], where $\zeta=\mu_{B} \sqrt{2 \pi} / p_{y}$ is the Casson parameter for the viscous-plastic fluid. To describe the MHD wave dynamics, we can apply Fourier analysis to Equation (2) and (8). For that purpose, applying the linearization to the Eq. (2) and (8) with 
respect to first order perturbation proportional to $\exp \left[-\iota\left(\omega t+k_{\perp} y+k_{\|} x\right)\right]$, where $\omega$ is the angular frequency, $k_{\perp}$ is the perpendicular component of the wave vector, and $k_{\|}$is the component of the wave vector along the magnetic field, the equations become,

$$
\begin{gathered}
\rho_{0}(-i \omega) u+\mu_{B}(1+ \\
\left.\frac{1}{\zeta}\right)\left(-i k_{\perp}\right)^{2} u-\frac{2 B_{0}^{2}}{\eta} u=K T\left(-i k_{\|}\right) n
\end{gathered}
$$

$$
n(-i \omega)+n_{0}\left(-i k_{\|}\right) u=0
$$

Where $\rho_{0}$ and $n_{0}$ are equilibrium mass and number densities, respectively. Putting the value of $n=-n_{0} k_{\|} u / \omega$ from Equation (10) to (9), we get;

$$
\begin{aligned}
& \rho_{0} \omega u+i \mu_{B}\left(1+\frac{1}{\zeta}\right) k_{\perp}^{2} u+i \frac{2 B_{0}^{2}}{\eta} u- \\
& \frac{n_{0} K T k_{\|}^{2}}{\omega} u=0
\end{aligned}
$$

The following relation can be found when rearranging above Equation (11).

$$
\begin{aligned}
& \quad \omega^{2}+i \frac{\mu_{B}}{\rho_{0}}\left(1+\frac{1}{\zeta}\right) k_{\perp}^{2} \omega+i \frac{2 B_{0}^{2}}{\eta \rho_{0}} \omega- \\
& C_{S}^{2} k_{\|}^{2}=0
\end{aligned}
$$

Here, $C_{s}^{2}=K T / m$ is the acoustic speed in the Casson fluid. The dimensionless form of above Equation (13) can be derived by defining dimensionless variables such as $\widetilde{\Omega}=\omega \tau_{A} \quad, S=\tau_{\eta} / \tau_{A}, \quad \operatorname{Pr}=\mu_{B} / \rho_{0} \tau_{A} \beta=$ $C_{S}^{2} / v_{A}^{2}$ (here, $v_{A}^{2}=B_{0}^{2} / 4 \pi \rho_{0}$ is the Alfven speed), while $\tau_{\eta}=\frac{L}{D_{m}}$ (with $D_{m}=\eta c^{2} /$ $4 \pi$ ), and $\tau_{A}=L / v_{A}$ are resistive diffusion (associated with magnetic field diffusion) and Alfven (associated with MHD wave) time scales respectively. Using above definitions, we get dimensionless form of above relation describing unstable MHD wave dynamics in Casson fluid flowing in the reverse roll for coating purpose;

$$
\beta k_{\|}^{2}=0
$$

For the first time, we have presented MHD wave dynamics for the non-Newtonian fluid. The relation might be called as viscousresistive-acoustic (VRA) mode, which is a polynomial having two roots.

\section{Results and Discussion}

The two roots of Equation (13) defining the dynamics of two MHD waves, one acoustic wave and the other Alfven wave, are coupled because of the presence of an externally applied magnetic field. The complex frequency $\Omega$ consists of two parts, real and imaginary. The real part describes only the propagation of acoustic wave in the fluid, while imaginary part narrates the growth in unstable coupled acoustic-Alfven wave. Acoustic waves are hydrodynamical propagation of disturbance through adiabatic compression and decompression that propagate energy across a fluid. While, the Alfvén wave is a MHD wave in which charged particles oscillate in response to a restoring force given by effective tension on magnetic field lines. The presence of a free energy source may result in oscillations that are unstable. In the current investigation, we 
found coupled unstable MHD wave dynamics are caused by magnetic energy. To estimate the growth rate of unstable modes we have plotted the imaginary part of the Equation (13) as a function of different controlling parameters of fluid dynamics in Figures 2 to 8 . Parallel and perpendicular dynamics are characterized in terms of propagation of parallel and perpendicular wave number with respect to magnetic field, which is extremely useful in fluid dynamics. We have solved the Eqn. (13) numerically for fluid dynamics controlling parameters, $S=10^{3},=10^{4}$, and $\zeta=1$.
Figure 2 displays the variation of the growth rate of the unstable mode against the parameter $\beta$, this parameter is actually describing the ratio of kinetic pressure of the charge fluid to magnetic pressure associated with the externally applied magnetic field. By keeping the perpendicular wave number fixed at $k_{\perp}=10$, increasing the wave number associated with parallel propagation causes a decrease in the growth of the unstable MHD wave. The dashed and dotted lines represent lower values of parallel wave number.

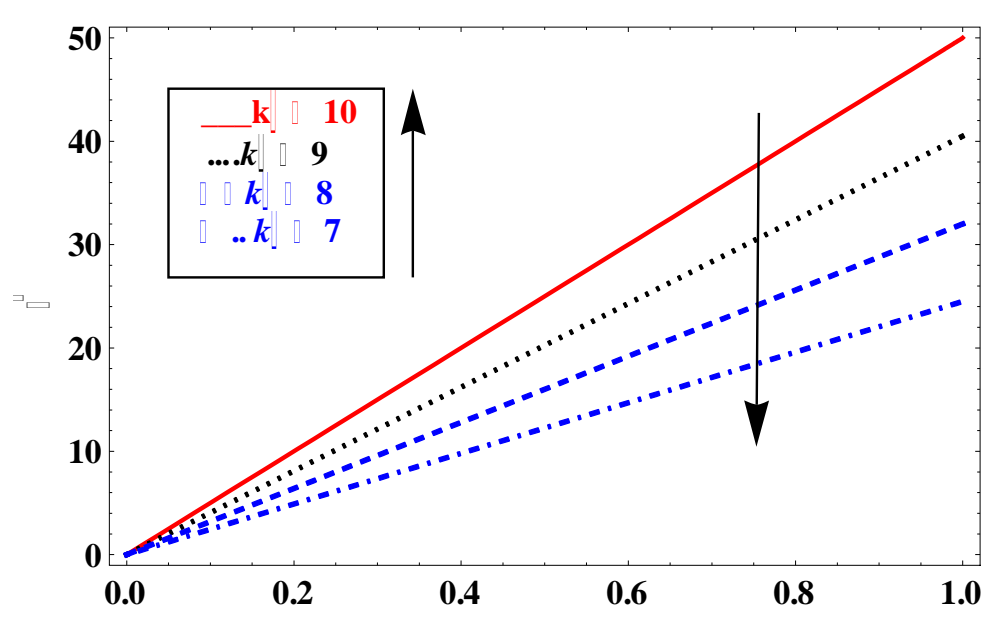

Figure 2. Variation of the growth rate of unstable MHD mode against fluid beta. 


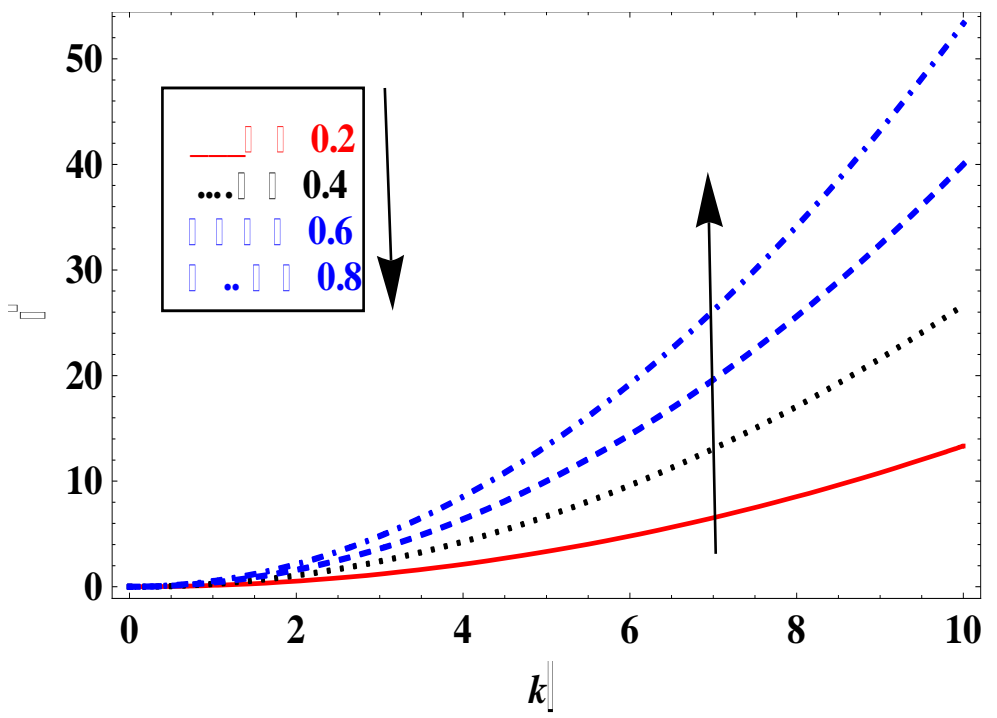

Figure 3. Variation of the growth rate against parallel wave number.

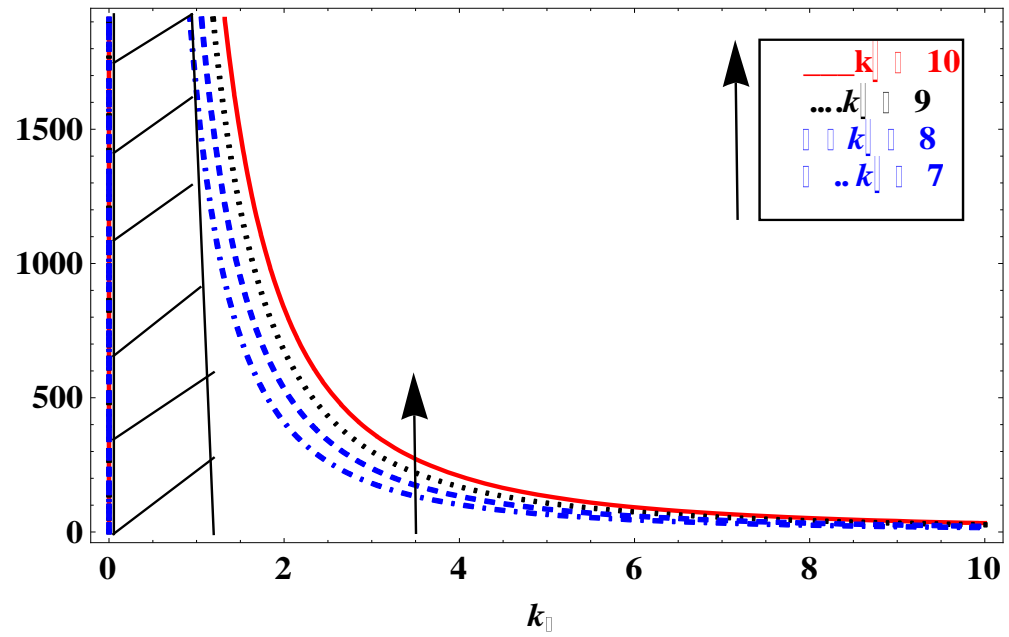

Figure 4. Variation of the growth rate of MHD mode against perpendicular wave number. 


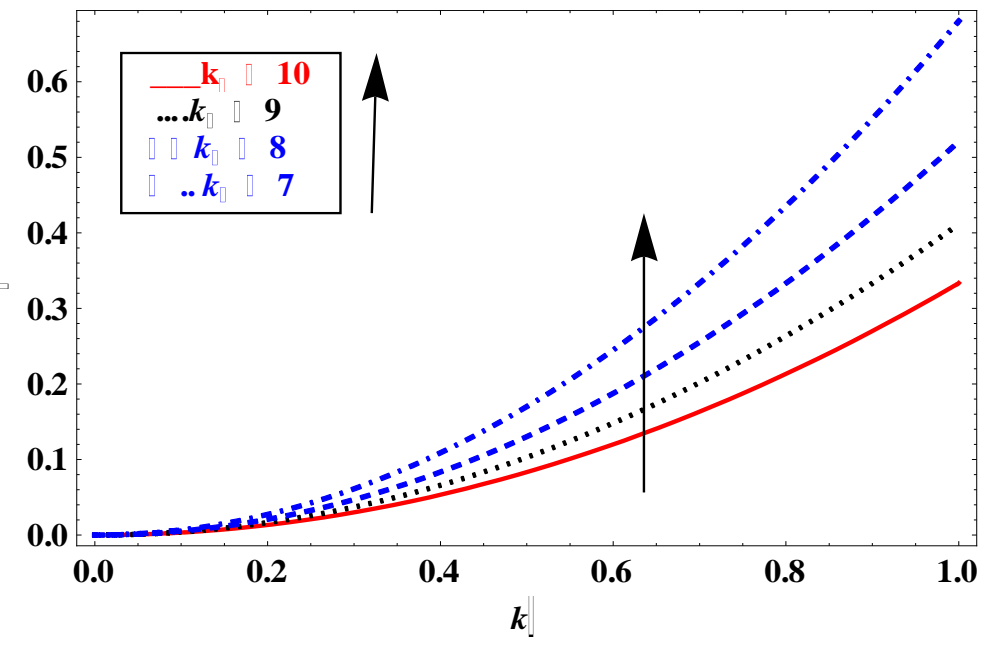

Figure 5. Variation of the growth rate of MHD mode against parallel wave number.

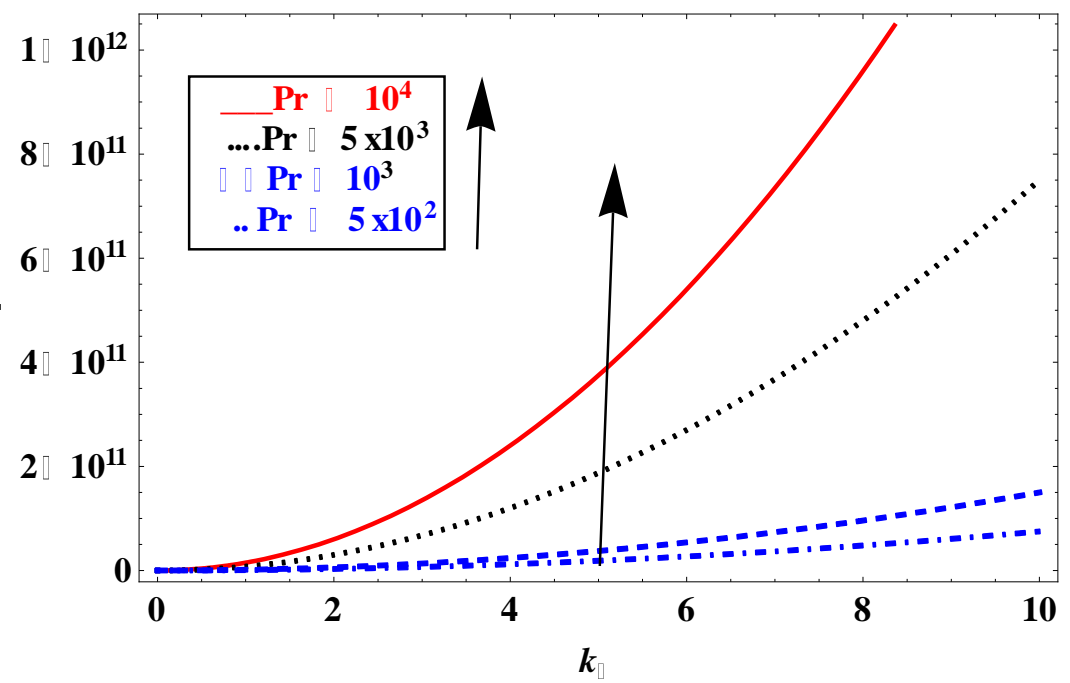

Figure 6. Variation of unstable MHD mode vs perpendicular wave number at different Pr. 


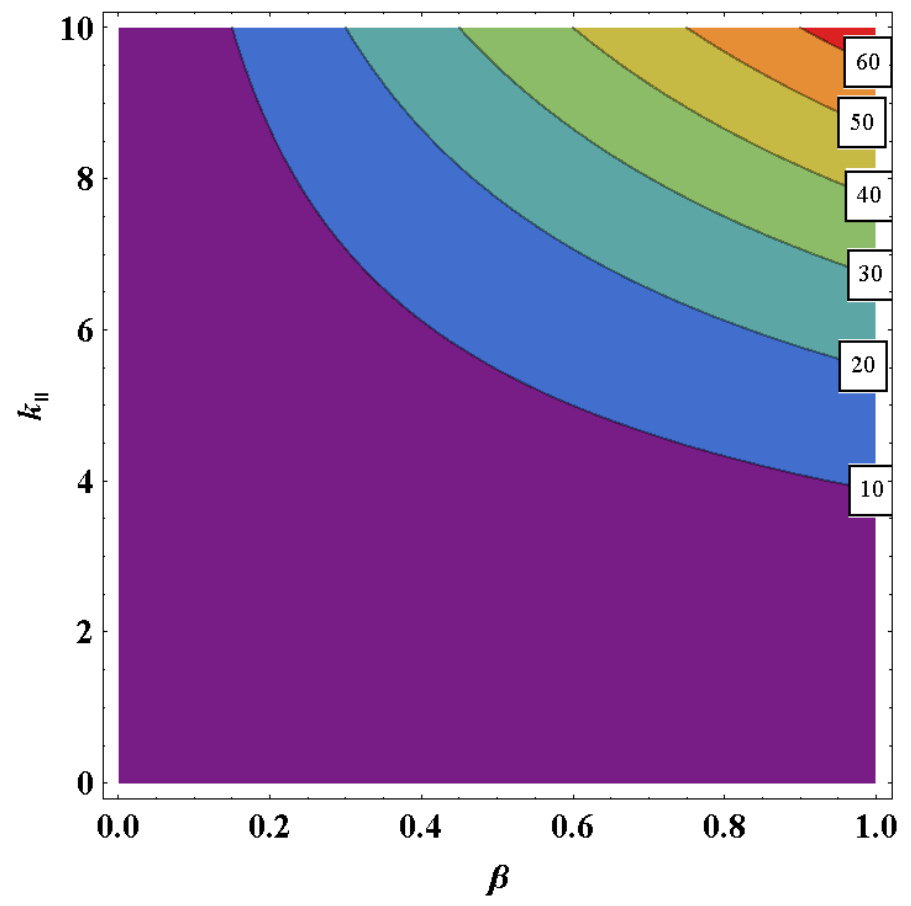

Figure 7. Contour plot of growth rate variation.

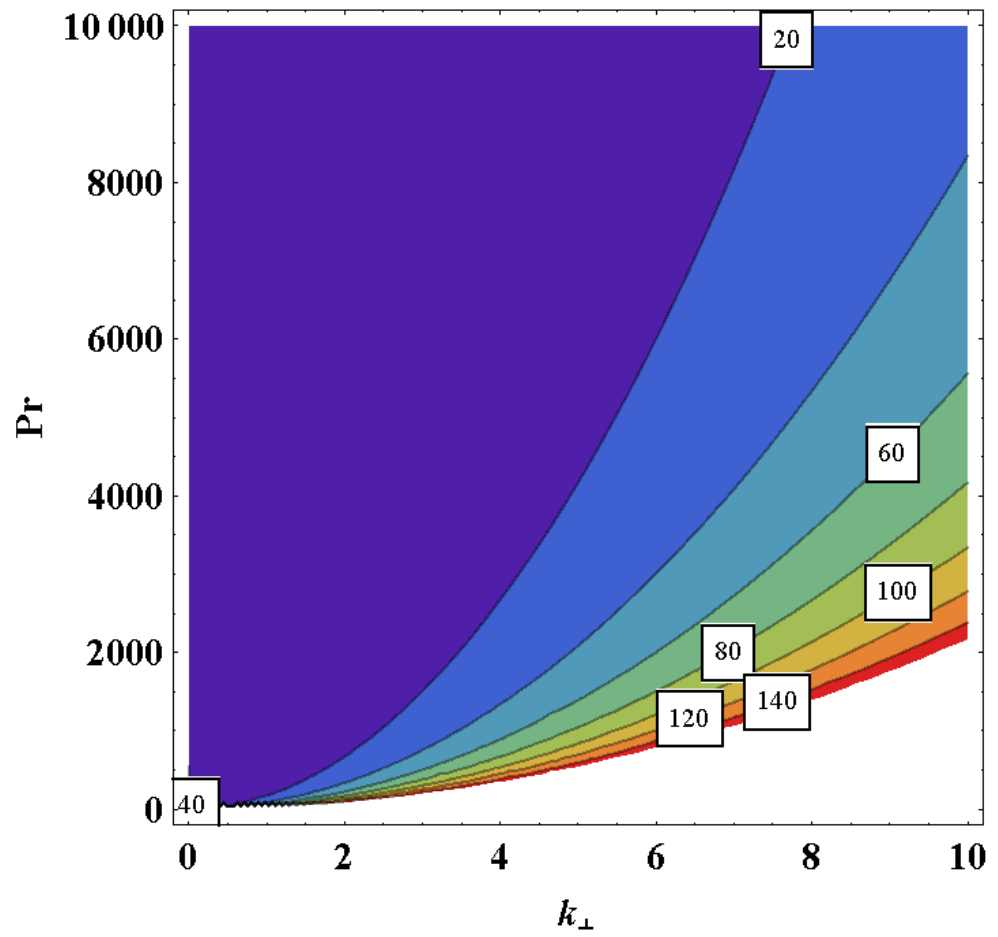

Figure 8. Contour plot of growth rate. 
In Figure 3, the variation of the growth rate of the unstable mode against the parallel wave number $k_{\|}$is plotted. The dependence of mode frequency follows acoustic wave $\widetilde{\Omega}=k_{\|}$at small $k_{\|}$ predominantly electrostatic in character. At large $k_{\|}$, frequency grows exponentially, indicating the electromagnetic nature of the mode, which is confirming the coupling of MHD waves. The controlling parameters are same as described in figure 2. Decreasing the value of the fluid beta $\beta$ is enhancing the growth of the unstable MHD wave due to increasing free energy in magnetic field. Figure 4 is the variation of the growth rate of the unstable mode against the perpendicular wave number $k_{\perp}$. Cut offs and resonances are found to the case of perpendicular propagation of MHD wave in the charged fluid flowing in the externally applied magnetic field. There are no propagation regions between 0 to 1 and resonance at $k_{\perp}=1$ is found. The effect of increasing the parallel wave number on the MHD mode's growth rate is investigated. The growth rate increases as the parallel wave number increase. Further, in figure 5, the growth rate of the MHD instability is plotted from the Eqn. (13), with the same fluid parameters (defined in in figure 1). Growth rate vs normalized parallel wave number are plotted for different values of perpendicular wave number $k_{\perp}$. The dominant unstable MHD mode found at lower values of perpendicular wave number. The most unstable MHD modes are found for the perpendicular wave number. Increasing the Prandtl number significantly enhance the growth of the unstable mode as shown in figure 6 .

The analytical relation (13) is now treated to parametric analysis in figure 7 and 8. The imaginary part of the unstable mode is numerically solved. The imaginary part of the relation represents the growth rate spectrum $\widetilde{\Omega}$ of the unstable mode. The growth rate shows non-monotonic behavior. Figure 7 and 8 are the contour plots of relation () and illustrates the variation of the growth rate with the fluid beta $\beta$ against normalized parallel wave number $k_{\|}$and normalized perpendicular wave number $k_{\perp}$, respectively. Plot shows growth rate dependence of the MHD mode on parallel and perpendicular wave-number at fixed Lundquist number $S=10^{3}$, Prandlt number $\operatorname{Pr}=10^{4}$ and visous-plastic fluid parameter $\zeta=1 \quad$ as the fluid beta $\beta$ increases the growth rate increases.

These images show that in the presence of a magnetic field, MHD wave dynamics are extremely relevant in charged particle based coatings. We have demonstrated MHD wave dynamics for the first time, including the viscosity effect of a non-Newtonian fluid. It's worth noting that the unstable MHD wave dynamics have a substantial impact on coating thickness stability via control parameters. Although fluid characteristics such as fluid velocity, coating thickness, pressure gradient distribution, flow rate, the graphical relationship between the coating thickness and the roll velocities ratio, and temperature distribution have previously been examined [23-24], none of the existing articles address $\mathrm{MDH}$ wave dynamics and instability conditions for such plasma-based processes. 


\section{Conclusion}

A mathematical model has been constructed and addressed to explore the fluid film flow of viscous-plastic fluids in the presence of a magnetic field in relation to the many uses of coating in engineering and industrial processes. The Casson fluid is taken into consideration in the flow model. Numerical techniques were used to solve the obtained equation.

The following are the work's main concluding remarks:

1. Given the simplicity of the lubrication model and the flow pattern in the region of the separation area, it may sound surprising that there is a fluid flow instability that affects coating thickness.

2. Numerically generated plots are used to investigate the effects of magnetic diffusion, viscous-plasticity, and MHD instabilities on the flow of Casson material.

3. The MHD instability has also been found to have a significant impact on the coating of Casson material.

4. The most unstable MHD mode is found at higher value of Prandtl number with respect to parallel propagation of the MHD wave.

Scope: Engineers and scientists from related industries throughout the world are invited to validate our findings in a practical situation. The theoretical MHD analysis of viscous-plastic materials was the focus of our research.

\section{References}

[1]. Balzarotti, F.; Rosen, M. Systematic study of coating systems with two rotating rolls. Lat. Am. Appl. Res. 2009, 39, 99-104.

[2].Zahid, M.; Zafar, M.; Rana, M.A.; Lodhi, M.S.; Awan, A.S.; Ahmad, B. Mathematical analysis of a non-newtonian polymer in the forward roll coating process. J. Polym. Eng. 2020, 40, 703-712.

[3].Zahid, M.; Zafar, M.; Rana, M.A.; Rana, M.T.A.; Lodhi, M.S. Numerical analysis of the forward roll coating of a rabinowitsch fluid. J. Plast. Film Sheeting 2020, 36, 191208.

[4]. Savage, M.D. Mathematical models for coating processes. J. Fluid Mech. 2006, 117, 443-455.

[5]. Greener, J.; Sullivan, T.; Turner, B.; Middleman, S. Ribbing instability of a tworoll coater: Newtonian fluids. Chem. Eng. Commun. 1980, 5, 73-83.

[6]. Chandio, S.; Webster, M. Numerical study of transient instabilities in reverse-roller coating flows. Int. J. Numer. Methods Heat Fluid Flow 2002, 12, 375-403.

[7]. G. Mahanta, S. Shaw, 3D Casson fluid flow past a porous linearly stretching sheet with convective boundary condition, Alexandria Engineering 2015, 54, 653-659.

[8]. M. Mustafa, J.A. Khan, Model for flow of Casson nanofluid past a non-linearly stretching sheet considering magnetic field effects, AIP Adv. 2015, 5077148.

[9]. I.L. Animasaun, E.A. Adebile, A.I. Fagbade, Casson fluid flow with variable thermophysical property along Exponentially stretching sheet with suction and exponentially decaying internal heat generation using the homotopic analysis Method, J. Niger. Math. Soc. 2015, 35, 1-17.

[10]. M. Das, R. Mahato, R. Nandkeolyar, Newtonian heating effect on unsteady hydro-magnetic Casson fluid flow past a flat 
plate with heat and mass transfer, Alexandria Eng. J. 2015, 54, 871-879.

[11]. N.V. Ganesh, B. Ganga, A.K.A. Hakeem Lie symmetry group analysis of magnetic field effects on free convective flow of a nano fluid over a semi-infinite stretching sheet J. Egypt. Math. Soc. 2014, 22, 304-310.

[12]. H. Dessie, N. Kishan MHD effects on heat transfer over stretching sheet embedded in porous medium with variable viscosity, viscous dissipation and heat source/sink Ain Shams Eng. J. 2014, 5, 967-977.

[13]. A.K.A. Hakeem, R. Kalaivanan, N.V. Ganesh, B. Ganga Effect of partial slip on hydro magnetic flow over a porous stretching sheet with non-uniform heat source/sink, thermal radiation and wall mass transfer Ain Shams Eng. J. 2014, 5, 913 922.

[14]. T. Hayat, M.S. Anwar, M. Farooq, A. Alsaedi, Mixed convection flow of viscoelastic fluid by a stretching cylinder with heat transfer, PLoS One 2015.

[15]. M.Y. Malik, T. Salahuddin, A. Hussain, S. Bilal MHD flow of tangent hyperbolic fluid over a stretching cylinder: using Keller box method J. Magn. Magn. Mater. 2015, 395, 271-276.

[16]. N. Sandeep, C. Sulochana, B. Rushi Kumar, Unsteady MHD radiative flow and heat transfer of a dusty nano fluid over an exponentially stretching surface, Eng. Sci. Technol. Int. J. 2016, 19 (1), 227-240.
[17]. Zafar, M.; Rana, M.A.; Zahid, M.; Ahmad, B. Mathematical analysis of the coating process over a porous web lubricated with upper-convected Maxwell fluid. Coatings 2019, 9, 458.

[18]. Zafar, M.; Rana, M.A.; Zahid, M.; Malik, M.A.; Lodhi, M.S. Mathematical analysis of roll coating process by using couple stress fluid. J. Nanofluids 2019, 8, 1683-1691.

[19]. Zheng, G.; Wachter, F.; Al-Zoubi, A.; Durst, F.; Taemmerich, R.; Stietenroth, M.; Pircher, P. Computations of coating windows for reverse roll coating of liquid films. J. Coat. Technol. Res. 2020, 17, 897910.

[20]. Ramos, J. J. General expression of the gyro viscous force Phys. Plasmas 2005, 12, 112301.

[21]. Chang, Z. and J. D. Callen Generalized gyro viscous force and its effect on the momentum balance equation. Phys. Fluids B 1992, 4, 1766-71.

[22]. Shercliff, J.A. Textbook of Magnetohydrodynamics; Pergamon Press: Oxford, UK, 1965.

[23]. Savage, M.D. Mathematical models for coating processes. J. Fluid Mech. 2006, 117, 443-455.

[24]. Fateh, A., Yanren H., Zahid M, and Rana M. A. Theoretical Study of the Reverse Roll Coating of Non-Isothermal Magnetohydrodynamics Viscoplastic Fluid. Coatings 2020, 10, 940.

\section{Creative Commons Attribution License 4.0 (Attribution 4.0 International, CC BY 4.0)}

This article is published under the terms of the Creative Commons Attribution License 4.0 https://creativecommons.org/licenses/by/4.0/deed.en US 